\title{
THE INFLUENCE OF TENSILE FORCES ON THE DEFLECTION OF CIRCULAR DIAPHRAGMS IN PRESSURE SENSORS
}

\section{J A VOORTHUYZEN and P BERGVELD}

Twente University of Technology, PO Box 217, 7500 AE Enschede (The Netherlands) (Recelved September 26, 1984, in revised form November 14, 1984, accepted November 23, 1984)

\section{Abstract}

It is known that the deflection of a diaphragm is determined by two mechanısms, bending moments or bending stress and tensile forces or membrane stress

Usually the influence of tensile forces is not taken into account when calculating the mechanical properties of thin diaphragms Hence the mathematical description thus obtained will only be valid if the deflection of the diaphragm is small compared with its thickness and if lateral stress is absent

In this paper we will consider uniformly loaded curcular diaphragms, which are assumed to be isotropic, and present the results of a study on the influence of bending stress and tensile stress, which together determine the diaphragm deflection

Starting from theoretical considerations, a simulation program is developed of which several results are presented and discussed

\section{Introduction}

Durnng recent years many papers have been presented concerning the development of pressure-sensitive semiconductor devices [1-14] All electronic detection mechanisms are based on the measurement of the deflection of a diaphragm as the result of a pressure difference across it In general this measurement is piezoresistive or capacitive

Many mechanical as well as electronic aspects of pressure sensors have been studied in detall $[9,11,12]$ which has increased the understanding of pressure-sensing mechanisms, but the influence of tensile forces on the deflection of circular diaphragms has not been studied in such a manner

Several authors $[2,11,13]$ have noted that packaging and thermal expansion introduce lateral forces which cause the deflection of a diaphragm to deviate from the behaviour calculated by using the generally accepted first-order theory, as for instance described by Timoshenko et al [15] 
It is known that the deflection of a diaphragm is determined by two mechanisms, normally described as bending moments or bending stress and tensile forces or membrane stress

Usually no account is taken of the influence of tensile forces in calculating the mechanical properties of thin diaphragms The mathematical description thus obtained will only be valid if the deflection of the diaphragm is small compared with its thickness and if lateral stress is absent [15]

Lee and Wise developed a rather complicated simulation program which considers the influence of lateral stress due to thermal loading [9], but up to now nobody has presented a more detaled study of these effects

It is interesting to note that Warren et al have studied the deflection of stretched curcular polymer membranes, on which they found that in this case the deflection is determined by membrane stress and that the influence of bending stress can be ignored [16,17]

So, for extreme cases, in which the influence of either bending stress or tensile stress can be ignored, the deflection of a curcular membrane or diaphragm can be described in a rather simple way, as has been done in the literature mentioned above

However, in many practical situations both mechanisms will affect the deflection at the same time, in which case we have to use a generalized and therefore more complicated mathematical description

In this paper we will consider uniformly loaded circular diaphragms, which are assumed to be isotropic, and present the results of a study concerning the influence of both bending and tensle stress, which together determine the diaphragm deflection

It will be clear that for anisotropic materials, the mathematical description presented below will have to be modified, as was described by Yasukawa et al [14] for example

In the following sections the mathematical description is presented and the boundary conditions are given Using the finite-difference numerical method, a set of non-linear equations is obtained, which can be solved by applying an iteration procedure

Starting from these considerations we have developed a simulation program of which several results are presented and discussed

\section{Mathematical description}

In order to achieve a mathematical description of the diaphragm deflection as a function of tensle stress, we will follow the method presented and discussed by Timoshenko et al [15] and will use the same notation

Here circular diaphragms will be considered having a radius $a$, a thickness $h$, Polsson's ratio $\nu$ and Young's modulus $E$ The coordinate centre $O$ is chosen at the centre of the undeflected curcular diaphragm and the radial distance of a point in the middle plane of the plate from the coordinate centre 
is denoted by $r$ The displacement in the radial direction of a point located in the middle plane of the diaphragm is written as $u$ and in the direction perpendicular to the plane of the undeflected diaphragm as $w$, as shown in Fig 1

The radial and tangential forces per unit length are written as $N_{\mathrm{r}}$ and $N_{\mathrm{t}}$ respectively It will be clear that generally $N_{\mathrm{r}}$ and $N_{\mathrm{t}}$ are both functions of the applied pressure $p$ In the case of zero pressure $p$, they are both equal to the initial tensile force per unit length which exists due to stretching of the diaphragm, packaging stress, thermal loadıng and so on, and is denoted as $N_{1}$

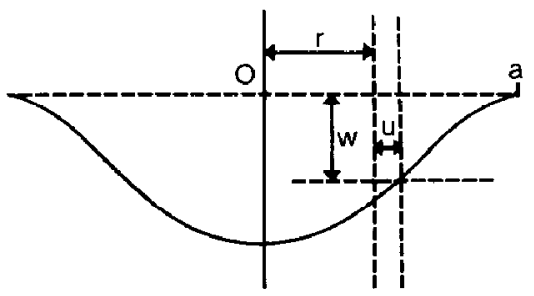

Fig 1 Cross section of a deflected circular diaphragm

In the case of small deflections or small initial stress, $N_{\mathrm{r}}$ and $N_{\mathrm{t}}$ are assumed to have little or no influence on the diaphragm deflection

Both forces and moments acting upon the diaphragm must be in equilibrium, in which case it can be shown that [15]

$\frac{\mathrm{d}^{3} w}{\mathrm{~d} r^{3}}+\frac{\mathrm{d}^{2} w}{r \mathrm{~d} r^{2}}-\frac{\mathrm{d} w}{r^{2} \mathrm{~d} r}=\frac{N_{\mathrm{r}}}{D} \cdot \frac{\mathrm{d} w}{\mathrm{~d} r}+\frac{p \cdot r}{2 D}$

$N_{\mathrm{r}}+\frac{r \mathrm{~d} N_{\mathrm{r}}}{\mathrm{d} r}-N_{\mathrm{t}}=0$

with $p$ the applied pressure difference across the diaphragm and $D$ the flexural rigidity, which is determined by

$D=\frac{E \cdot h^{3}}{12\left(1-\nu^{2}\right)}$

Equations (1) and (2) comprise three unknowns, namely $N_{\mathrm{r}}, N_{\mathrm{t}}$ and $w$, hence we need a third equation to be able to solve the set of equations

In the case of zero initial stress such a relation has already been derived, and relates membrane stresses and diaphragm deflections [15] Using the same fundamental description as given in [15], it can be shown that this relation can also be used for non-zero values of the initial tensile force $N_{1}$ per unit length The radial and tangential strain, which are functions of the deformation of the diaphragm, are denoted as $\epsilon_{\mathrm{r}}$ and $\epsilon_{\mathrm{t}}$ respectively

For zero pressure $p$ and non-zero values of the initial tensile force $N_{1}$ per unit length, $\epsilon_{\mathrm{r}}$ and $\epsilon_{\mathrm{t}}$ will be equal to the initial strain $\epsilon_{\mathrm{i}}$

The radial strain $\epsilon_{\mathrm{r}}$ also depends on the unit elongation in the radial durection and therefore we can write [15] 
$\epsilon_{\mathrm{r}}-\epsilon_{1}=\frac{\mathrm{d} u}{\mathrm{~d} r}+\frac{1}{2}\left(\frac{\mathrm{d} w}{\mathrm{~d} r}\right)^{2}$

Analogously the tangential strain $\epsilon_{t}$ is a function of the unit elongation in the tangential direction, and can be written as

$\epsilon_{\mathrm{t}}-\epsilon_{\mathrm{i}}=u / r$

Applying Hooke's law, the tangential and radial strains can be related to the tangential and radial forces per unit length

$\epsilon_{\mathrm{r}}=\left(N_{\mathrm{r}}-\nu N_{\mathrm{t}}\right) / h \cdot E$

$\epsilon_{\mathrm{t}}=\left(N_{\mathrm{t}}-\nu N_{\mathrm{r}}\right) / h \cdot E$

Using relations (2) and (4)-(7), we obtam an expression that relates diaphragm deflections and tensile forces per unit length

$r \cdot \frac{\mathrm{d}\left(N_{\mathrm{r}}+N_{\mathrm{t}}\right)}{\mathrm{d} r}+\frac{h E}{2} \cdot\left(\frac{\mathrm{d} w}{\mathrm{~d} r}\right)^{2}=0$

This result is also found for zero values of the initial tensile force per unit length [15]

For the sake of simplicity, the first derivative of the displacement $w$ will be written as

$f=\mathrm{d} w / \mathrm{d} r$

Using this result, the order of eqn (1) is reduced If we substitute eqn (2) into eqn (8) and also use eqn (9), $f$ can be calculated by simultaneously solving the following set of second-order differential equations

$\frac{\mathrm{d}^{2} f}{\mathrm{~d} r^{2}}+\frac{\mathrm{d} f}{r \cdot \mathrm{d} r}-\frac{f}{r^{2}}=\frac{N_{\mathrm{r}} \cdot f}{D}+\frac{p \cdot r}{2 D}$

$\frac{r^{2} \mathrm{~d}^{2} N_{\mathrm{r}}}{\mathrm{d} r^{2}}+\frac{3 r \mathrm{~d} N_{\mathrm{r}}}{\mathrm{d} r}=\frac{-h E}{2} \cdot f^{2}$

Considering the differential eqns (10) and (11), which are of the second order, it can be concluded that they can be solved only if two boundary conditions are known for $N_{\mathrm{r}}$ as well as for $f$

If the edge of the circular diaphragm is clamped, the first derivative of the displacement $w$ is equal to zero at this edge

The displacement $w$ is a symmetrical function of the radial distance $r$, which means that the first derivative is also equal to zero at the centre of the diaphragm The boundary conditions are therefore

$f(0)=f(a)=0$

Using relations (2), (5) and (7) the radial displacement $u$ can be written exclusively as a function of $N_{\mathrm{r}}$ 
$u=\frac{r}{E h}\left((1-\nu) \cdot\left(N_{\mathrm{r}}-N_{1}\right)+r \frac{\mathrm{d} N_{\mathrm{s}}}{\mathrm{d} r}\right)$

Note that this result is obtained by making use of the fact that if the radial and tangential forces per unit length are equal to the initial value $N_{1}$, the radial and tangential strains will be equal to the initial value $\epsilon_{1}$

It will be clear that the radial displacement $u$ vanıshes at the edge of the diaphragm, and thus we obtain

$\frac{\mathrm{d} N_{\mathrm{r}}(a)}{\mathrm{d} r}=\frac{(1-\nu)}{a} \cdot\left(N_{1}-N_{\mathrm{r}}(a)\right)$

At the same time $N_{\mathrm{r}}$ will be a symmetrical function of the radial distance $r$, which implies that

$\mathrm{d} N_{\mathrm{r}}(0) / \mathrm{d} r=0$

If $f$ has been calculated using eqns $(10)$ and (11) and boundary conditions (12), (14) and (15), the displacement $w$ can finally be found by integrating $f$

$w(r)=-\int_{a}^{r} f(r) \mathrm{d} r$

assuming $w$ is equal to zero at the edge of the diaphragm

\section{Solution procedure}

The non-linear character of eqns (10) and (11) makes it difficult to find an analytical expression for the unknowns $f$ and $N_{\mathrm{r}}$

For this reason we have decided to approach the solution of the differential equations by applying the finite difference numencal method, combined with an iteration procedure

The interval $[0, a]$ of the radial distance $r$ is divided into $n$ identical smaller intervals with length $\Delta r=a / n$

At every node corresponding to these intervals, the differential equations can be replaced by finite difference equations $[18,19]$ The approxImated values of $N_{\mathrm{r}}$ and $f$ at the $l$ th node are denoted as $N_{\mathrm{r}}[l]$ and $f[l]$ respectively Using the Taylor series expansion and numbering the nodes equivalent to the current value of $r(r=\imath \cdot \Delta r)$, we obtain the following equations

$$
\begin{aligned}
& \left(2 \imath^{2}-\imath\right) \cdot f[\imath-1]+\left(-4 \imath^{2}-2-N_{\mathrm{r}}[\imath] \cdot \frac{2 \imath^{2} \Delta r^{2}}{D}\right) \cdot f[l]+ \\
& \quad+\left(2 \imath^{2}+\imath\right) \cdot f[\imath+1]=\frac{p \imath^{3} \Delta r^{3}}{D}
\end{aligned}
$$


$\left(l^{2}-\frac{3}{2} l\right) \cdot N_{\mathrm{r}}[l-1]+\left(-2 l^{2}\right) \cdot N_{\mathrm{r}}[l]+\left(l^{2}+\frac{3}{2} l\right) \cdot N_{\mathrm{r}}[l+1]=\frac{-h E f[l]^{2}}{2}$

in which case it can be shown that the error of the approximated results $f[l]$ and $N_{\mathrm{r}}[l]$ compared to the exact solutions will be proportional to the square of the step-size $\Delta r$

Dealing with the boundary conditions as formulated above in an analogous way to obtain eqns (17) and (18), we acquire two sets of nonlinear equations, which can be denoted in an abridged form as

$A\left(\mathrm{~N}_{\mathrm{r}}\right) \cdot \mathrm{f}=B(p)$

$C \cdot \mathrm{N}_{\mathrm{r}}=D(\mathbf{f})$

where $A$ is a matrix whose elements, being a function of the radial force $N_{\mathrm{r}}$ per unit length, can be found by using the left side of eqn (17)

$B$ is a vector that is a function of pressure $p$, as can be seen from the right side of eqn (17), and $f$ and $N_{r}$ are vectors that contain the elements $f[l]$ and $N_{\mathrm{r}}[l]$ respectively

In the same way the elements of matrix $C$ are obtained by using the left side of eqn (18), while vector $D$, which is a function of $f$, can be derived from the right side of this equation

To solve the non-linear eqns (19) and (20) we have used the following iteration procedure

We have furst chosen reasonable values for the elements of the vector $\mathrm{N}_{\mathrm{r}}$, after which the elements of matrix $A$ can be calculated Using these results the vector $f$ can be found by solving eqn (19), after which the vector $\mathrm{N}_{\mathrm{r}}$ can be calculated by eqn (20) Using these results the elements of matrix $A$ can be calculated and so on

If the values of $\mathbf{N}_{x}$ and $\mathbf{f}$ in the $k$ th step of the iteration procedure are written as $\mathbf{N}_{\mathrm{r}}^{k}$ and $\mathbf{f}^{k}$ respectively, the iteration procedure can be described by

$$
\begin{aligned}
& A\left(\mathrm{~N}_{\mathrm{r}}^{k}\right) \mathrm{f}^{k+1}=B(p) \\
& C \cdot \mathrm{N}_{\mathrm{r}}^{k+1}=D\left(\mathrm{f}^{k+1}\right)
\end{aligned}
$$

It will be clear that the iteration procedure must be stopped as soon as the required accuracy has been achieved As an approximation of the error, the relative difference $\epsilon$ between two calculated results in succeeding steps of the iteration process is commonly used [20] For this reason the procedure can be terminated if

$$
\left|\frac{f[l]^{k+1}-f[l]^{k}}{f[l]^{k+1}}\right|<\epsilon
$$

in which case $\iota$ must have a fixed value

Finally the deflection $w$ of the diaphragm can be calculated by applying a numerical integration method to approach the exact solution as defined by eqn (16) We have used the trapezoidal rule, in which case we can write 
$w[l]=w[l+1]-\frac{\Delta r}{2}(f[l+1]+f[\imath])$

It can be shown that the error of the approximated results compared with the exact solution will also be proportional to the square of the step-size $\Delta r$ [19]

\section{Simulation results}

Starting from the theory described above, we have developed a rather simple simulation program which calculates the mechanical behaviour of circular diaphragms and accounts for the influence of tensile forces

The results will be valid for initially stretched membranes, as described by Warren et al [16, 17], as well as for strongly deflected diaphragms, which up to now have not been discussed in detail in the literature

In our simulations we have used curcular diaphragms with an outer radius $a$ of $1 \mathrm{~mm}$ and a thickness of $25 \mu \mathrm{m}$, whle Poisson's ratio is assumed to be equal to 03 The relative difference $\epsilon$ between the results of two succeeding iteration steps, as defined by eqn (23) is chosen to be $10^{-3}$, which is sufficiently small for this study We have chosen the number of intervals $n$ to be equal to 40 , which corresponds to a step size $\Delta r$ of $25 \mu \mathrm{m}$

To solve the matrix equations we have applied standard subroutines of the NAG-library, which use the so-called LU-decomposition

Figure 2 shows the calculated centre deflection $w(0)$, normalized to the thickness $h$, as a function of the pressure $p$, normalized to Young's modulus $E$, with the initial stress per unit length $N_{1}$, relative to $E \cdot h$ as a parameter

It can be shown that using these normalizations the presented results will be valid for all different diaphragms with the same ratio between radius $a$ and thickness $h$, which in this case is 40

Curve A of Fig 2 describes the centre deflection of the diaphragm if the influence of tensile forces is ignored, in which case the analytical solution of eqn (1) is given by

$w(r)=\frac{p}{64 D} \cdot\left(a^{2}-r^{2}\right)^{2}$

which result is commonly used in papers dealing with pressure sensors

Curve $B$ is the calculated centre deflection obtained by using a secondorder energy method approach [15], which gives more accurate results for strongly deflected diaphragms, of course after assuming that the initial tensile forces are equal to zero

Curves $\mathrm{C}$ to $\mathrm{H}$ represent the calculated centre deflection as a function of pressure by applying the simulation program discussed above for $N_{1} / E \cdot h$ equal to $0,2 \times 10^{-4}, 4 \times 10^{-4}, 10 \times 10^{-4}, 20 \times 10^{-4}, 40 \times 10^{-4}$, respectively

Note that for small deflections curves $A, B$ and $C$ coincide, which is in accordance with the fact that in this case the influence of tensile forces can be ignored 

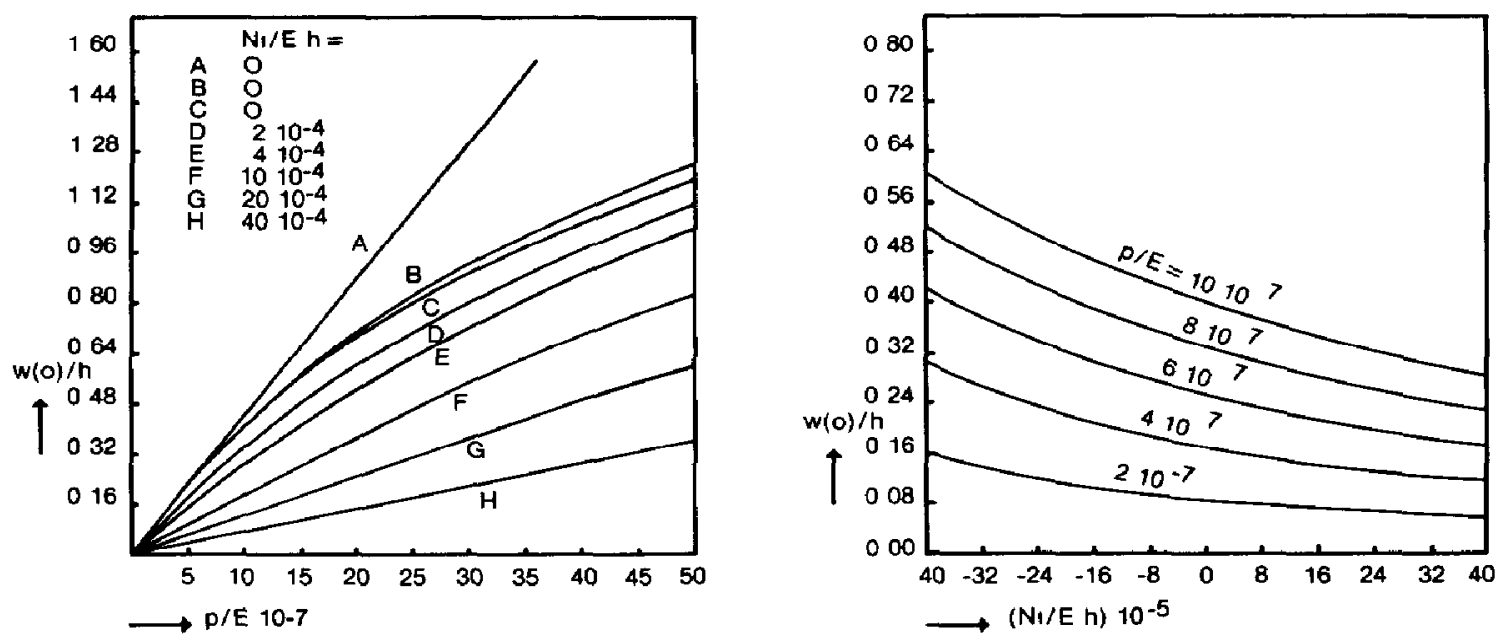

Fig 2 Centre deflection as a function of pressure, with the initial tensile force per unit length as a parameter, using conventional models (curve A, B) and the present model under various conditions of initial tensile force (curve $\mathrm{C}$ to $\mathrm{H}$ )

Fig 3 Centre deflection as a function of initial tensile force per unit length, with the normalized pressure as a parameter, using the present model

Considering curves $\mathrm{B}$ and $\mathrm{C}$ we can conclude that the second-order approach, as presented in the literature [15], describes the actual centre deflection for zero initial stress with an accuracy that is better than 95 per cent for the given pressure range

It can be seen from curves $\mathrm{C}$ to $\mathrm{H}$ that the diaphragm deflection decreases for increasing values of the initial force per unit length, as can also be seen from Fig 3

This Figure represents the centre deflection of the diaphragm as a function of the initial force per unit length $N_{1}$, with the applied pressure as a parameter, using the same normalizations as in Fig 2

Considering the results presented in Fig 3 it can be concluded that the centre deflection varies by about a factor of two over the given range of the initial force $N_{1}$ per unit length

An indication of the practical meaning of these theoretical results can be given by the following example

Let us assume that a diaphragm is mounted on a supporting backplate and that the mismatch in thermal expansion coefficients between diaphragm and support is equal to $10^{-6}{ }^{\circ} \mathrm{C}$, which is the case if an $\mathrm{S}_{1}$ diaphragm is mounted on a piece of glass $[10,11]$ Using the theoretical considerations of Section 2 , it can be shown that the initial strain $\epsilon_{1}$ is a direct measure of the normalized tensile force $N_{1} / E h$

If the rigidity of the backplate is taken as infinite as compared to the rigidity of the diaphragm, a temperature change of $100^{\circ} \mathrm{C}$ will introduce a strain of $10 \times 10^{-5}$, which implies a normalized tensile force $N_{1} / E \cdot h$ of about $10 \times 10^{-5}$ 
It will be clear that due to the presence of a supporting rim and the fact that the rigidity of the supporting blackplate is not infinite, as discussed by Yasukawa et al [14] for example, the actual value of $N_{1} / E \cdot h$ will be smaller than the value calculated above of $10 \times 10^{-5}$ Considering this calculated value and the simulation results presented in Fig 3, the temperature coefficlent of diaphragm deflection is estimated to be $1000 \mathrm{ppm} /{ }^{\circ} \mathrm{C}$, which corresponds rather well with the value of $275 \mathrm{ppm} /{ }^{\circ} \mathrm{C}$ measured for a $24 \mu \mathrm{m}$ thick square draphragm [10]

Note that in the case of negative values of the lateral force, it is more usual to talk about compressive forces rather than tensile forces In this respect we have to point out that the existence of compressive forces can lead to mechanically unstable behaviour of the diaphragm [21]

For small negative values of $N_{1}$ a pressure difference across the diaphragm will cause a small deflection, which disappears if this pressure is removed However, increasing negative values of $N_{1}$ create a situation in which a relatively small pressure difference produces a relatively large deflection, which remains when the pressure is diminished In the literature an expression has been derived for the critical value of $N_{1}$ above which unstable behaviour, usually known as buckling, wlll occur [21]

$-\left(N_{1}\right)_{\mathrm{crit}}=\frac{1468 E h^{3}}{12\left(1-\nu^{2}\right) a^{2}}$

This implies that in our simulations the critical value of the normalized parameter $-N_{1} / E \cdot h$ is equal to $84 \times 10^{-4}$

Other simulations, which will not be presented here, have shown that the calculated diaphragm deflection really does increase rapidly for values of $-N_{1} / E \cdot h$ above a value of $80 \times 10^{-4}$

Therefore we conclude that the simulation results presented in Fig 3, with a maximum value of $40 \times 10^{-4}$, are not influenced by buckling effects In practice, however, it will be clear that we have to be aware that improper mechanical handling of a pressure sensor can still involve buckling effects which are fatal for sensor operation

In the rest of this paper we will not consider the case of compressive forces and will restrict ourselves to the influence of tensile forces only

Warren et al $[16,17]$ have studied the mechanical properties of stretched polymeric membranes, which have a thermal expansion coefficient of the order of $10^{-4} /{ }^{\circ} \mathrm{C}$ It will be clear that in this case we can obtain much higher values for the normalized parameter $N_{1} / E \cdot h$

Figure 4 shows results obtained by using the simulation program for higher values of $N_{1} / E \cdot h$

If we assume $N_{\mathrm{r}}$ and $N_{\mathrm{t}}$ to be constant and equal to $N_{1}$ and if we ignore the influence of the third denvative of $w$, which means that the influence of bending moments can be neglected, the solution of eqn (1) is given by

$w(r)=\frac{p}{4 N_{1}} \cdot\left(a^{2}-r^{2}\right)$ 

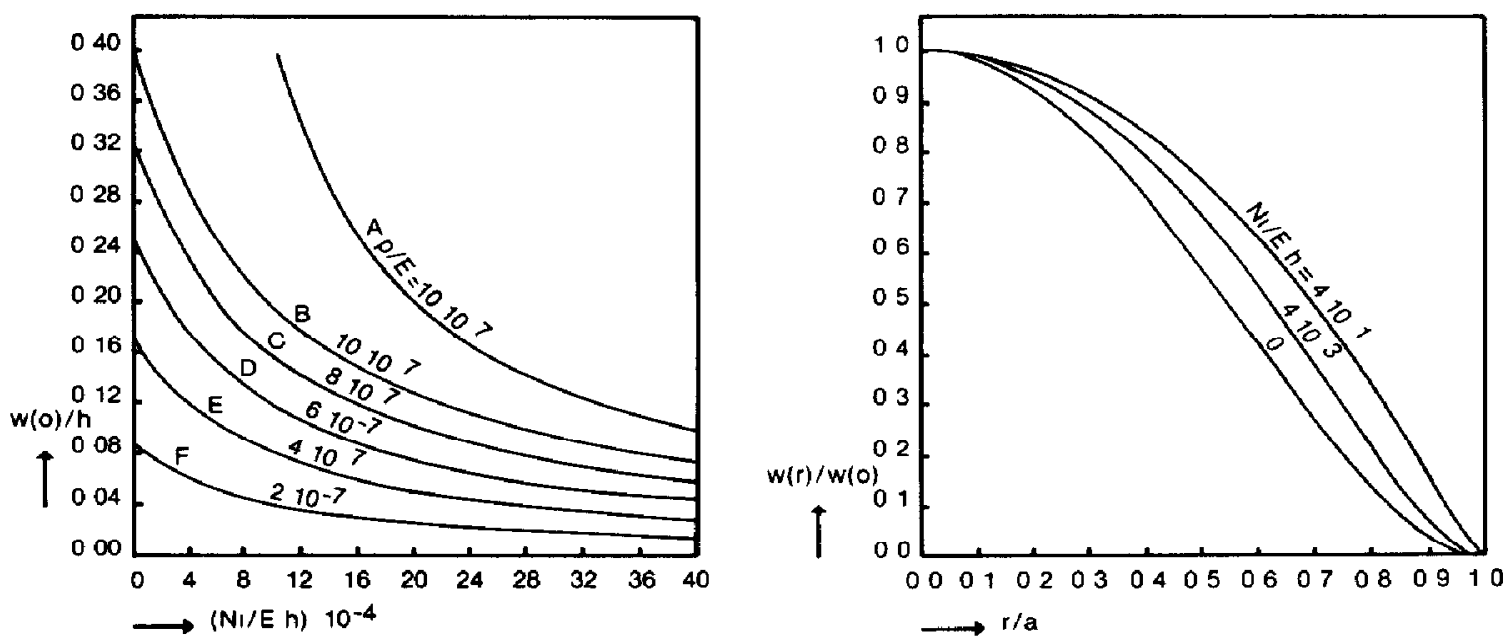

Fig 4 Centre deflection as a function of large initial tensile forces, with the normalized pressure as a parameter, using eqn (27) (curve A) and the present model (curve B to F)

Fig 5 Diaphragm deflection as a function of the radial distance, with $N_{1} / E h$ as a param eter and $p / E=10^{-6}$, using the present model

This result is also used by Warren et al to calculate the deflection of stretched membranes

The calculated centre deflection as a function of $N_{1} / E$ by applying eqn (27) is given by curve A of Fig 4 All other curves of Fig 4 are obtained by using the simulation program

Considering these results, we can conclude that eqn (27) will be valid only for very large values of $N_{1} / E \cdot h$

We also see that the deflection of the diaphragm strongly depends on the initial tensile forces for small values, and that this dependence is not linear

It will be clear that for purposes of sensor design we are not only interested in the centre deflection of the diaphragm, but also in the shape of the deflected diaphragm, $\imath e$, in the deflection as a function of the radial distance $r$

In Fig 5 the calculated deflection $w(r)$, normalized to the centre deflection $w(0)$, is presented for $p / E$ equal to $10^{-6}$ and three different values of $N_{1} / E \cdot h$, namely $0,4 \times 10^{-3}$ and $4 \times 10^{-1}$ These results are obtained by using the simulation program

Considering Fig 5 we can say that the shape of the deflected diaphragm for small values of $N_{1} / E \cdot h$ and $p / E$ behaves as a fourth-order expression like eqn (25), and for very large values of $N_{1} / E \cdot h$ as a second-order expression like eqn (27)

Figure 6 depicts the diaphragm deflection, normalized to its thickness $h$, as a function of the radial distance $r$ for $p / E$ equal to $10^{-6}$ and $N_{1} / E \cdot h$ equal to $4 \times 10^{-2}$

Curve $A$ is obtained by using the simulation program and curve $B$ by applying eqn (27) Note that for this value of $N_{1} / E \cdot h$ the two curves $\mathrm{A}$ and 

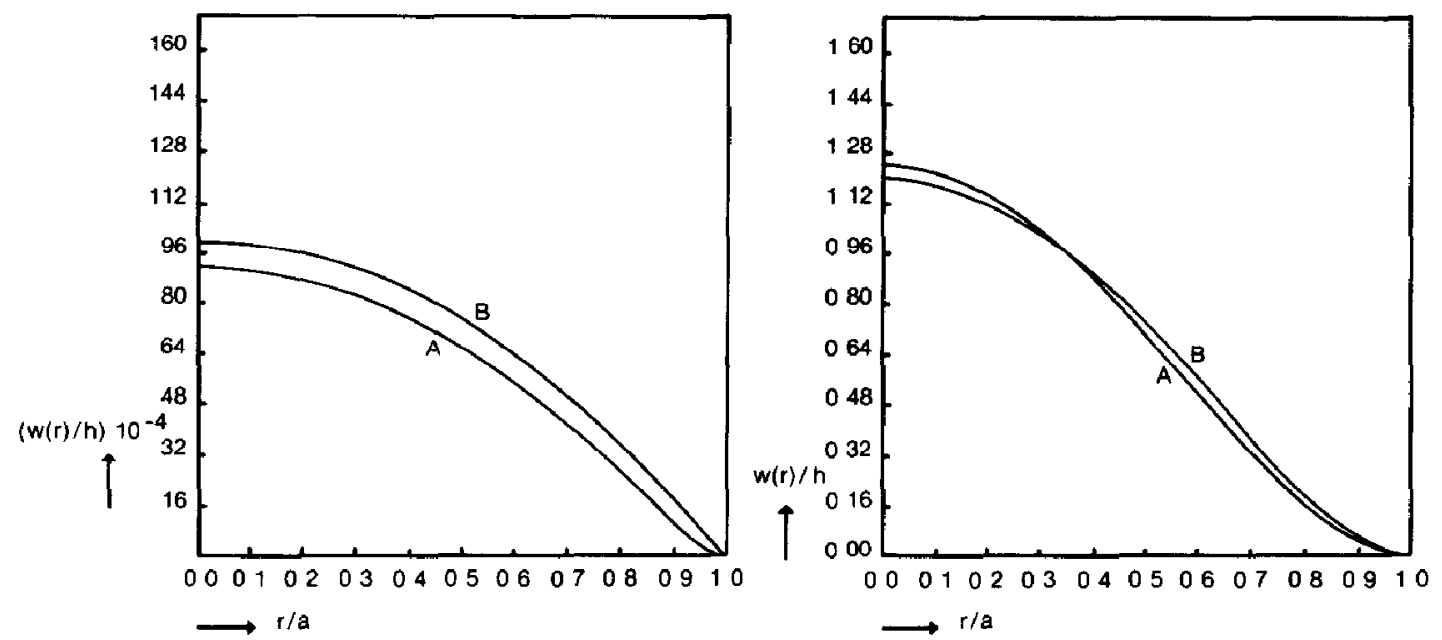

Fig 6 Diaphragm deflection as a function of the radial distance, for $p / E=10^{-6}$ and $N_{1} / E h=4 \times 10^{-2}$, using the present model (curve A) and eqn (27) (curve B)

Fig 7 Diaphragm deflection as a function of the radial distance, for $p / E=5 \times 10^{6}$ and $N_{1} / E h=0$, using a second -order energy method approach (curve $\mathbf{A}$ ) and the present model (curve B)

B are almost parallel to each other At the centre of the diaphragm the relative difference between the two curves is less than 10 per cent, but the relatıve difference increases for larger values of $r / a$

In Fig 7 we have drawn the diaphragm deflection normalized to its thickness $h$, as a function of the radial distance $r$ for $p / E$ equal to $5 \times 10^{-6}$ and $N_{1} / E \cdot h$ equal to zero

Curve $A$ is achieved by using the second-order energy method approach mentioned above [15], and curve $B$ by using our simulation program Considering these results we can conclude that in the case of zero initial stress, the centre deflection as well as the shape of the diaphragm are very well described by curve $A$ Note that the value of $p / E$ used is relatively large for the pressure sensors described in the literature [1 - 4], which implies that in many reasonable situations the differences between the two curves will be smaller than is shown in Fig 7

\section{Conclusions}

Starting from the mathematical description presented in the literature, we have developed a simulation program which accounts for the influence of tensile forces on the mechanical properties of curcular diaphragms

In this way it has been possible to calculate the behaviour of a strongly deflected diaphragm and to study the influence of initial lateral forces on the deflection

Using a realistic example, we have shown that because of these forces the real deflection can differ by about a factor of two (Fig 3) 
The simulated results for extreme cases are in very good agreement with the results presented in the literature [15-17], which suggests the reliability of the results obtained by using our simulation program

\section{Acknowledgement}

The authors wish to thank Professor C de Pater for his valuable advice and helpful comments on the preparation of this paper

\section{References}

$1 \mathrm{~S} K$ Clark and K D Wise, Pressure sensitivity in anisotropically etched thindiaphragm pressure sensors, IEEE Trans Electron Devices, ED 26 (12) (1979) 1887 1896

2 W $H$ Ko, J Hynecek and $\mathbf{S}$ F Boettcher, Development of a miniature pressure transducer for biomedical applications, IEEE Trans Electron Devices, ED-26 (12) (1979) $1895-1905$

3 K Yamada, M Nishihara, $R$ Kanzawa and $R$ Kobayashi, A piezoresistive integrated pressure sensor, Sensors and Actuators, 4 (1983) $63-69$

$4 \mathrm{P} J$ French and A $P$ Dorey, Frequency output plezoresistive pressure sensor, Sensors and Actuators, 4 (1983) $77-83$

5 S Sugıyama, M Takıgawa and I Igarashı, Integrated pıezoresistıve pressure sensor with both voltage and frequency output, Sensors and Actuators, 4 (1983) 113120

$6 \mathrm{Y}$ Kanda, Optımum desıgn considerations for silıcon pressure sensors using a fourterminal gauge, Sensors and Actuators, 4 (1983) $199-206$

7 W H Ko, B X Shao, C O Fung, W -J Shen and G -J Yeh, Capacitive pressure transducers with integrated circuits, Sensors and Actuators, 4 (1983) $403-411$

$8 \mathrm{C} M$ Lawson and $\mathrm{V} J$ Tekippe, Fiber-optic diaphragm curvature pressure transducer, Opt Lett, 8 (1983) $286-288$

$9 \mathrm{~K}-\mathrm{W}$ Lee and $\mathrm{K} \mathrm{D}$ Wise, SENSIM a simulation program for solid-state pressure sensors, IEEE Trans Electron Devices, ED 29 (1982) $34-41$

$10 \mathrm{Y} S$ Lee and $\mathrm{K} D$ Wise, $A$ batch-fabricated silicon capacitive pressure transducer with low temperature sensitıvity, IEEE Trans Electron Devices, ED 29 (1982) 42 . 48

$11 \mathrm{~W} H$ Ko, $\mathrm{M}-\mathrm{H}$ Bao and $\mathrm{Y}-\mathrm{D}$ Hong, A high-sensitive integrated circuit capacitive pressure transducer, IEEE Trans Electron Devices, ED 29, (1982) 48 - 56

12 K Yamada, M Nishıhara, S Shimada, M Tanabe, M Shimazoe and Y Matsuoka, Nonlinearity of the piezoresistance effect of p-type silicon diffused layers, $I E E E$ Trans Electron Devices, ED 29 (1982) 7177

$13 \mathrm{M}$ Esashı, H Komatsu, $\mathbf{T}$ Matsuo, $M$ Takahashı, $\mathbf{T}$ Takıshıma, $\mathbf{K}$ Imabayashı and $H$ Ozawa, Fabrication of catheter-tıp and sidewall miniature pressure sensor, IEEE Trans Electron Devices, ED-29 (1982) $57 \quad 63$

14 A Yasukawa, S Shımada, Y Matsuoka, Y Kanda, Design consideratıons for silıcon circular diaphragm pressure sensors, Jpn J Appl Phys, 21 (1982) $1049-1052$

$15 \mathrm{~S}$ Timoshenko and $\mathrm{S}$ Woinowski-Krieger, Theory of Plates and Shells, 2nd edn, McGraw-Hill, New York, 1959

$16 \mathrm{~J}$ E Warren, A M Brzezınski and J F Hamilton, Capacitance-microphonc static membrane deflections, $J$ Acoust Soc Am, 52 (1972) $711-719$

17 J E Warren, Capacitance-microphone static membrane deflections comments and further results, $J$ Acoust Soc Am, 58 (1975) $733-740$

$18 \mathrm{~J} A \mathrm{~N}$ Lee, Numerical Analysis for Computers, Reinhold Publ Corp, New York, 1966 
$19 \mathrm{R}$ W Southworth and S L Deleeuw, Digital Computation and Numerical Methods, McGraw Hill, New York, 1965

$20 \mathrm{R}$ Beckett and J Hurt, Numerical Calculations and Algorithms, McGraw-Hill, New York, 1967

21 S P Timoshenko and J M Gere, Theory of Elastic Stabulity, 2nd edn, McGraw-Hill, New York, 1961

\section{Biographes}

Johannes Arle Voorthuyzen was born in Capelle a/d IJssel, the Netherlands, on April 22, 1959 He recelved the M S degree in electrical engineering from the Delft University of Technology, Delft, The Netherlands, in 1982 In the same year he joined the Bio-information Group, Department of Electrical Engineering, Twente University of Technology, where he is working towards his $\mathrm{Ph} D$ degree $\mathrm{H} 1 \mathrm{~s}$ current research is focused on electromechanical sensors for biomedical applications

Plet Bergueld was born in Oosterwolde, the Netherlands, on January 26, 1940 He received the M S degree in electrical engineenng (electronics) from Eindhoven University of Technology, Eindhoven, the Netherlands, in 1965, and the Ph D degree from Twente University of Technology, Enschede, the Netherlands, in 1973

Since 1965 he has been a member of the Bio-information Group, Department of Electrical Engineering, Twente University of Technology $\mathrm{He}$ is also a member of the Coordination Centre for Biomedical Engineering and a member of the Research Unit, Sensors and Actuators, of this University In 1981 he became a member of the Semiconductor Group of the Foundation for Fundamental Research on Matter in the Netherlands

The subject of his dissertation was the ion-sensitive field-effect transistor (ISFET) and the OSFET He is involved in research on electronic measuring and stimulating methods in physiological systems with special attention on in vivo biosensors He lectures on biomedical instrumentation for graduate students of Twente University of Technology and medical personnel of affiliated hospitals

In 1984 he was appointed as full professor in biosensor technology at the Twente University of Technology 